\section{Maxillary sinus augmentation procedures through equine-derived biomaterial or calvaria autologous bone: immunohistochemical evaluation of OPG/RANKL in humans}

\author{
S. Tetè, ${ }^{1}$ R. Vinci, ${ }^{2}$ V.L. Zizzari, ${ }^{3}$ S. Zara, ${ }^{3}$ \\ V. La Scala, ${ }^{4}$ A. Cataldi, ${ }^{3}$ E. Gherlone, ${ }^{2}$ \\ A. Piattelli' \\ 'Department of Medical, Oral and \\ Biotechnological Sciences, University \\ "G. d'Annunzio", Chieti, Italy; \\ "Department of Oral Science, University \\ "Ateneo Vita - Salute” S. Raffaele, Milan, \\ Italy; \\ ${ }^{3}$ Department of Pharmacy, University \\ “G. d'Annunzio”, Chieti, Italy \\ ${ }^{4}$ Private practice, Pistoia, Italy
}

\section{Abstract}

Autologous bone is considered the gold standard for bone regeneration, even if different heterologous bone substitutes have been proposed to overcome the limits related to its use. The aim of this study was to analyze and to compare the molecular events switched on by autologous or heterologous bone graft insertion, focusing on TGF $\beta 1$ expression and OPG/RANKL ratio, to analyze resorption process, and estimating graft vascularization, new bone tissue deposition and its mineralization, through VEGF, BSP and SPARC expression evaluation, respectively. Patients needing pre-prosthetic rehabilitation of the posterior maxilla were treated using an equine-derived biomaterial (Group 1) or calvaria autologous bone (Group 2), according to the morphology of the bone defect. Bone graft integration was evaluated on bone samples obtained from the treated areas at the moment of dental implant insertion, by morphological and immunohistochemical analyses for TGF $\beta 1$, OPG, RANKL, VEGF, BSP, and SPARC expression. Morpho logical analysis shows the presence of biomaterial residual granules in Group 1, in parallel to a good integration between graft and host tissue. Moderate TGF $\beta 1$ expression is seen in both Group 1 and Group 2. OPG/RANKL ratio appears higher in Group 1; VEGF expression appears very strong in Group 1 and strong in Group 2, while BSP and SPARC expression results weak in Group 1 and moderate in Group 2. Our results reveal the good integration between both types of graft and the host tissue, even though autologous graft seems to produce a faster regenerative process, as evidenced by the different expression of the investigated molecules. According to these observations, the clinical use of heterologous particulate equine-derived biomaterial may ensure long-term predictability of implantprosthetic rehabilitation, comparable to that obtained with autologous bone graft.

\section{Introduction}

The use of regenerative procedures before implant-prosthetic oral rehabilitation is required to counteract vertical and horizontal bone loss and, mostly, to obtain an adequate bone quantity and quality to ensure primary stability at implant insertion. Several bone grafting materials were proposed for bone regeneration, such as autogenous, allogenic and xenogenic biomaterials, or a combination of them and, as the biological response of host tissue can be related to biomaterial origin, attention was focused on the interactions occurring between grafts and host tissue. ${ }^{1,2}$ As already reported by Mangano et al., heterologous biomaterials were found to be as clinically efficient as autologous bone for their capacity of osteoconduction, even if little is known about their capability to be resorbed, and about the time they need to be fully replaced by newly formed bone tissue. ${ }^{3}$ In fact, a biomaterial showing a too rapid resorption rate may result unsuitable for bone augmentation procedures, because it could be completely resorbed before osteogenic cells have colonized the defect; on the other hand, a biomaterial showing no resorption could cause problems to the regeneration of bone with a lower osteogenesis ability in respect to native autologous bone. ${ }^{4,5}$

In literature, only a few studies were reported about the use of an equine-derived bone substitute, which seems to be able to induce osteoblast differentiation, to be resorbed in vitro by osteoclasts and to be successfully used in mandibular ridge augmentation.,7 Moreover, the integration of a bone graft results in a remodeling process similar to the physiological bone healing event following a bone fracture. ${ }^{8}$ Bone remodeling occurs through different steps, which start from a lesion of the vascular structure at the site of the injury reducing the supply of nutrients and determining an initial bone resorption. The hematoma generated activates signaling molecules and growth factors, which stimulate proliferation and differentiation of osteo-progenitor cells. Among them, Transforming Growth Factor- $\beta$ (TGF- $\beta$ ) superfamily and angiogenic factors are included. In particular, TGF $\beta 1$ is essential for osteoblastic differentiation of mesenchymal precursors, it induces the synthesis of bone morphogenenetic proteins (BMPs) and promotes osteoid and extracellular protein production such as collagen, osteopontin and osteonectin. ${ }^{9}$ Moreover, TGF- $\beta 1$ is
Correspondence: Dr. Susi Zara, Department of Pharmacy, University "G. d'Annunzio", via dei Vestini 31, 66100 Chieti Scalo (CH), Italy. Tel/Fax: +39.0871.3554507.

E-mail: s.zara@unich.it

Key words: osteoconduction, OPG/RANKL ratio, biomaterial resorption, integration bone graft, calvaria, equine-derived biomaterial

Acknowledgments: the authors wish to thank FIRB-Accordi di Programma 2010 Project Processi degenerativi dei tessuti mineralizzati del cavo orale, impiego di biomateriali e controllo delle interazioni con $i$ microrganismi dell'ambiente, Prof A. Cataldi, for the fellowship attributed to Dr. V. L. Zizzari.

Received for publication: 12 November 2012. Accepted for publication: 29 December 2012.

This work is licensed under a Creative Commons Attribution NonCommercial 3.0 License (CC BYNC 3.0).

(C) Copyright S. Tetè et al., 2013

Licensee PAGEPress, Italy

European Journal of Histochemistry 2013; 57:e10 doi:10.4081/ejh.2013.e10

an important factor for osteoclasts and osteoblasts coupling: in fact, it not only promotes the recruitment of hematopoietic osteoclast precursors, but also shows an inhibitory effect on bone resorption and stimulates the production of osteoprotegerin (OPG). ${ }^{10}$

OPG is expressed by osteoblasts and regulates bone homeostasis by inhibiting osteoclastogenesis and bone resorption. ${ }^{11}$ OPG, binding to RANKL on osteoblast/stromal cells, blocks the RANKL/RANK interaction between osteoblast/stromal cells and osteoclast precursors, inhibiting osteoclast precursor differentiation, reducing osteoclast production and regulating osteoclast-mediated resorption. ${ }^{12}$ Moreover, in such processes a main role is also played by angiogenic factors, among which Vascular Endothelial Growth Factor (VEGF) is included. VEGF is produced by endothelial cells and osteoblasts and is involved in early bone remodeling phases since it controls osteoblast growth. ${ }^{13}$

The early phases of bone graft integration are followed by remodeling phenomena driven by molecules produced by osteoblasts, as Bone Sialoprotein (BSP) and Secreted Protein Acidic and Rich in Cysteine (SPARC), leading to new extracellular matrix deposition and to its subsequent mineralization. BSP is a component of the extracellular matrix which plays important functions in the regulation of new bone apposition and remodeling. BSP expression is up-regulated by factors inducing osteoblast differentiation, released by active osteogenic cells at the site of new bone formation, even though it has also been shown to 
promote osteoclastic resorption of mineralized surfaces. ${ }^{14,15}$

SPARC, also known as osteonectin, is a collagen-binding glycoprotein that appears to regulate cell growth through interactions with extracellular matrix (ECM) and cytokines. ${ }^{16}$ It is secreted by osteoblasts during bone formation, initiating a mineralization process and promoting mineral crystal formation. It is a modulator of the mineralizing process, essential for bone graft integration, such as the bone healing around dental implants. ${ }^{17}$

Based on this knowledge, the aim of our work was to analyze and to compare the molecular events switched on by autologous or heterologous bone graft insertion, focusing our attention on TGF $\beta 1$ expression and OPG/RANKL ratio, to analyze resorption process, and to estimate bone graft vascularization, new bone formation and its mineralization, through VEGF, BSP and SPARC expression evaluation, respectively. By understanding the mechanism underlying graft integration and by comparing the results obtained with the use of the heterologous bone substitute to that obtained with the use of autologous bone it should be verified if equinederived bone substitute possesses characteristic, which may permit a good integration within the host bone tissue, thus clinically ensuring an adequate primary stability to the implant and predictability of the implant-prosthetic rehabilitation.

\section{Materials and Methods}

\section{Patients selection}

Twenty patients (13 males, 7 females; age ranging 45-58) with inadequate bone volume in the posterior maxilla, were scheduled for bone augmentation procedures followed by implant placement. The patients were divided into two groups $(n=10)$, according to the severity and morphology of the bone defect, mirroring Classes $\mathrm{C}$ and $\mathrm{F}$ of the Chiapasco's Classification of the Posterior Maxilla (Table 1). ${ }^{18}$ All patients received sufficient information about the inclusion in this study and gave written consent in accordance with Italian Legislation and with the code of Ethical Principles for Medical Research involving Human Subjects of the World Medical Association (Declaration of Helsinki). Ten patients ( 8 males, 2 females; age ranging 45 54), having Class $\mathrm{C}$ bone defects, underwent maxillary sinus augmentation procedure with a bone substitute of equine origin (BioBone ${ }^{\circledR}$ Osteoconductor Mix, BioSAF IN S.r.l., Ancona, Italy) (Group 1), and ten patients (5 males, 5 females; age ranging 46-58), with Class $\mathrm{F}$ bone defects, received an onlay bone graft and a maxillary sinus augmentation procedure with bone obtained from the parietal region of the calvaria (Group 2).

BioBone $^{\circledR}$ Osteoconductor Mix is a reasorbable, collagen-deprived, and deantigenated osteoconductive biomaterial, obtained after a biological process of deantigenation at $37^{\circ} \mathrm{C}$ in a humid atmosphere, made up of a corticocancellous mixture of equine origin (particles width $0.5-1 \mathrm{~mm}$ ). The autologous bone blocks from the parietal region of calvaria were taken from calvaria under general anesthesia by a piezoelectric instrument (Easy Surgery ${ }^{\circledR}$, BioSAF IN S.r.l.), shaped according to the dimension of the defects, properly fitted in the recipient site, and fixed with lag screw to rebuild the alveolar ridge. All gaps between the bone blocks and the recipient sites and the maxillary sinus were packed with bone chips obtained from the same donor site, and the grafted areas covered with a resorbable barrier (Biobone $^{\circledR}$ Collagen Membrane, BioSAF IN S.r.l.)

Post-operative healing was uneventful for all the patients, and therefore, after about 6 months they were scheduled for a second surgery for implant placement. Contextually the intervention of implant insertion, bone samples were retrieved by a $3 \mathrm{~mm}$-diameter and 8 $\mathrm{mm}$-height trephine bur under sterile saline solution irrigation in the sites of implant placement, in order to obtain significant specimens of bone regenerated with heterologous bone substitute in Group 1 patients, while in Group 2 patients bone samples were obtained from the lateral maxillary wall between the sites of implant placement.

\section{Light microscopy analysis and immunohistochemistry}

Samples of bone tissues were fixed in $10 \%$ phosphate-buffered formalin for $24 \mathrm{~h}$, and decalcified in $10 \%$ tetrahydrated EDTA, according to data sheet (MIELODEC kit, Bio-Optica, Milan, Italy). Subsequently, they were dehydrated through ascending alcohols and xylene, and then paraffin embedded. Samples were dewaxed (xylene and alcohol at progressively

Table 1. Chiapasco's classification of the posterior maxilla.

\begin{tabular}{|c|c|c|c|}
\hline Class & $\begin{array}{c}\text { Height } \\
\text { of the residual } \\
\text { ridge }\end{array}$ & $\begin{array}{l}\text { Thickness of } \\
\text { the residual } \\
\text { ridge }\end{array}$ & Interarch distance \\
\hline A & $>5 \mathrm{~mm}<8 \mathrm{~mm}$ & $\geq 6 \mathrm{~mm}$ & Normal \\
\hline B & $>5 \mathrm{~mm}<8 \mathrm{~mm}$ & $<6 \mathrm{~mm}$ & Normal \\
\hline $\mathrm{C}$ & $<5 \mathrm{~mm}$ & $\geq 6 \mathrm{~mm}$ & Normal \\
\hline D & $<5 \mathrm{~mm}$ & $<6 \mathrm{~mm}$ & Normal \\
\hline E & $>5 \mathrm{~mm}<8 \mathrm{~mm}$ & $\geq 6 \mathrm{~mm}$ & Increased \\
\hline $\mathrm{F}$ & $>5 \mathrm{~mm}<8 \mathrm{~mm}$ & $<6 \mathrm{~mm}$ & Increased \\
\hline G & $<5 \mathrm{~mm}$ & $\geq 6 \mathrm{~mm}$ & Increased \\
\hline $\mathrm{H}$ & $<5 \mathrm{~mm}$ & $<6 \mathrm{~mm}$ & Increased \\
\hline
\end{tabular}
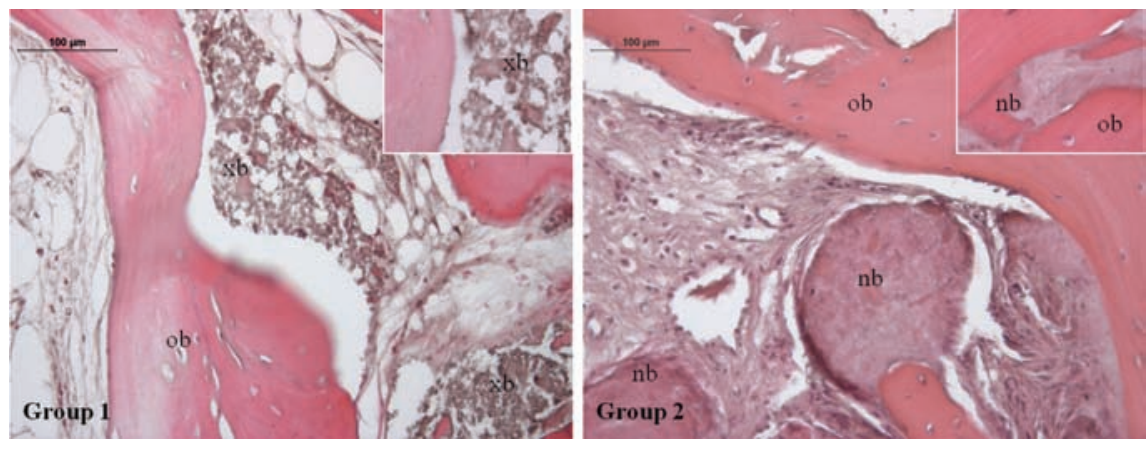

Figure 1. Hematoxylin and eosin staining of Group 1 and Group 2 specimens. Magnification 20x. Group 1: bone tissue specimens obtained from equine-derived bone substitute grafted area; Group 2: bone tissue specimens obtained from calvaria bone grafted area. Inset (40x) shows, in group 1 specimens, grafted biomaterial particles, in group 2 specimens, large mineralized areas within which newly formed bone can be recognized because of osteocyte lacunae lack and absence of lamellar organization; $x b$, xenogenous bone; ob, old bone; nb, new bone. 
decreasing concentrations), sliced $5 \mu \mathrm{m}$ thick and processed for hematoxylin-eosin staining and for immunohistochemical analysis.

In order to detect TGF $\beta 1$, OPG, RANKL, VEGF, BSP, and SPARC proteins, immunohistochemistry was performed on $5 \mu \mathrm{m}$-thick sections by means of Ultravision LP Detection System HRP Polymer \& DAB Plus Chromogen (Lab Vision Thermo, CA, USA). To reduce nonspecific background staining due to endogenous peroxidase, sections were incubated with hydrogen peroxidase block solution for $10 \mathrm{~min}$. Slides were then incubated in the presence of mouse anti-TGF $\beta 1$, anti-RANKL and antiSPARC monoclonal antibodies, and rabbit antiVEGF polyclonal antibody (Santa Cruz Biotechnology, Santa Cruz, CA, USA), mouse anti-OPG monoclonal antibody (Acris Antibodies, Herford, Germany), and mouse anti-BSP monoclonal antibody (Calbiochem, Darmstadt, Germany).

Sections were incubated in the presence of specific HRP-conjugated secondary antibodies. Peroxidase was developed using diaminobenzidin chromogen (DAB), and nuclei were hematoxylin counterstained. Negative controls were performed by omitting the primary antibody. Randomly selected slides belonging to each sample were then observed by means of Leica DM 4000 light microscopy (Leica Cambridge Ltd, Cambridge, UK), equipped with a Leica DFC 320 camera (Leica Cambridge Ltd) for computerized images.

\section{Computerized morphometry measurements and image analysis}

After digitizing the images obtained from the immunohistochemical stained sections, QWin Plus 3.5 software (Leica Cambridge Ltd) was used to evaluate TGF $\beta 1$, OPG, RANKL, VEGF, BSP and SPARC expression. Image analysis of protein expression was performed through the quantification of immunohistochemical brown chromogen, expressed as percentage of positive area respect to total area of the field, as an average value per ten fields, randomly chosen, for each sample at light microscope observation.

Moreover, intensity of staining (IS) was graded on a scale of $0-4$, according to the following assesssments: 0 , no detectable staining; 1 , weak staining; 2 , moderate staining; 3 , strong staining; 4 , very strong staining, as previously reported. ${ }^{19}$ The positive immunolabeling for TGF $\beta 1$, OPG, RANKL, and VEGF was cytoplasmic; the positive immunolabeling for BSP and SPARC was in the pericellular space.

Quantification of immunohistochemical brown chromogen was performed at $20 \mathrm{x}$ magnification by three different researchers, and the final result was a mean value of the three separate evaluations. Cohen's kappa coefficient was applied to measure the agreement between the three observers and averaged over all three to evaluate overall agreement using
A
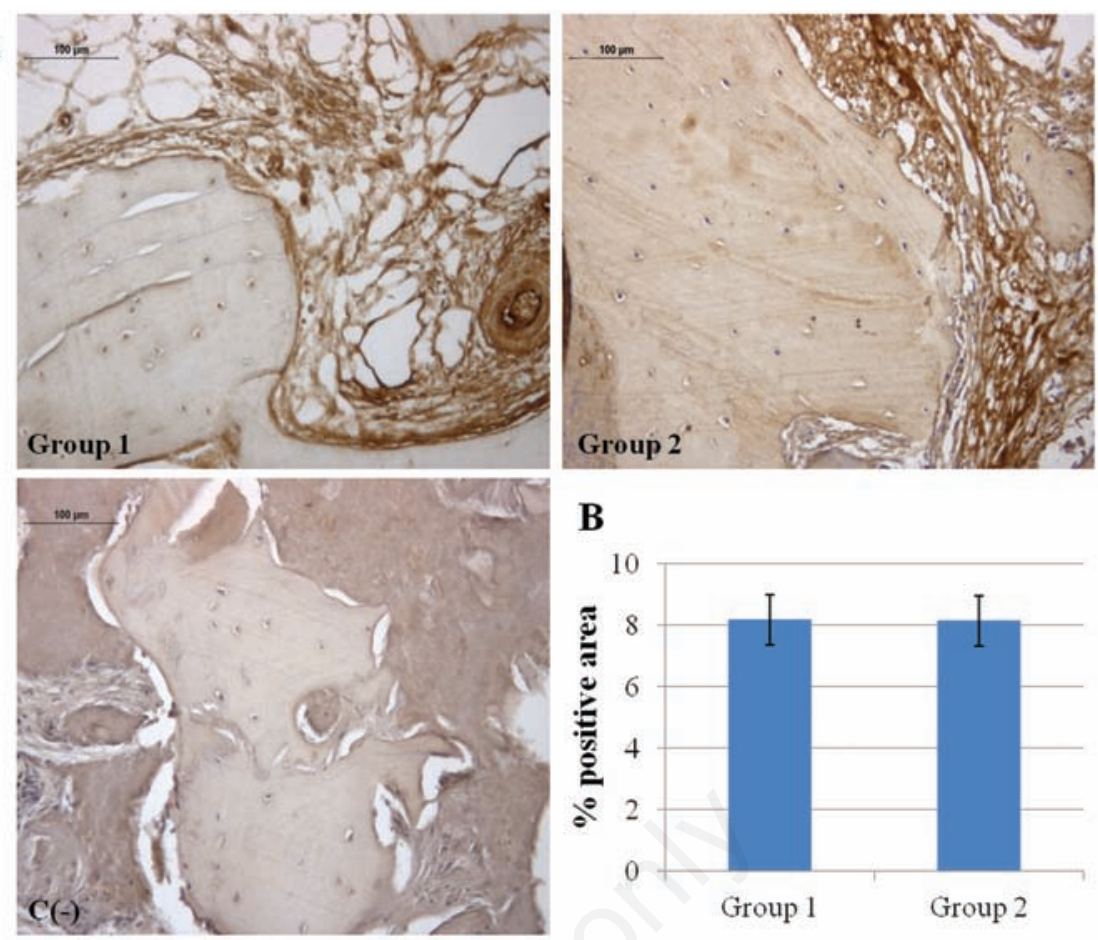

B

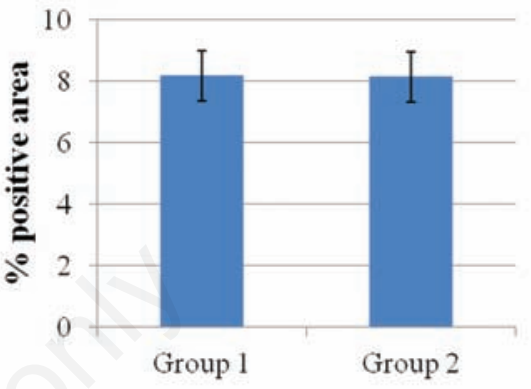

Figure 2. A) Immunohistochemical analysis of TGF $\beta 1$ expression in Group 1 and Group 2 specimens. Magnification 20x. Group 1: bone tissue specimens obtained from equinederived bone substitute grafted area; Group 2: bone tissue specimens obtained from calvaria bone grafted area; C(-), negative control. Moderate TGF $\beta 1$ immunolabeling of both Group 1 and Group 2 bone tissue; no TGF $\beta 1$ immunostaining is seen in negative control. B) Graphic representation of densitometric analysis of TGF $\beta 1$ positive area $\pm S D$, determined by direct visual counting of ten fields (mean values) for each of five slides per specimens at 20x magnification.

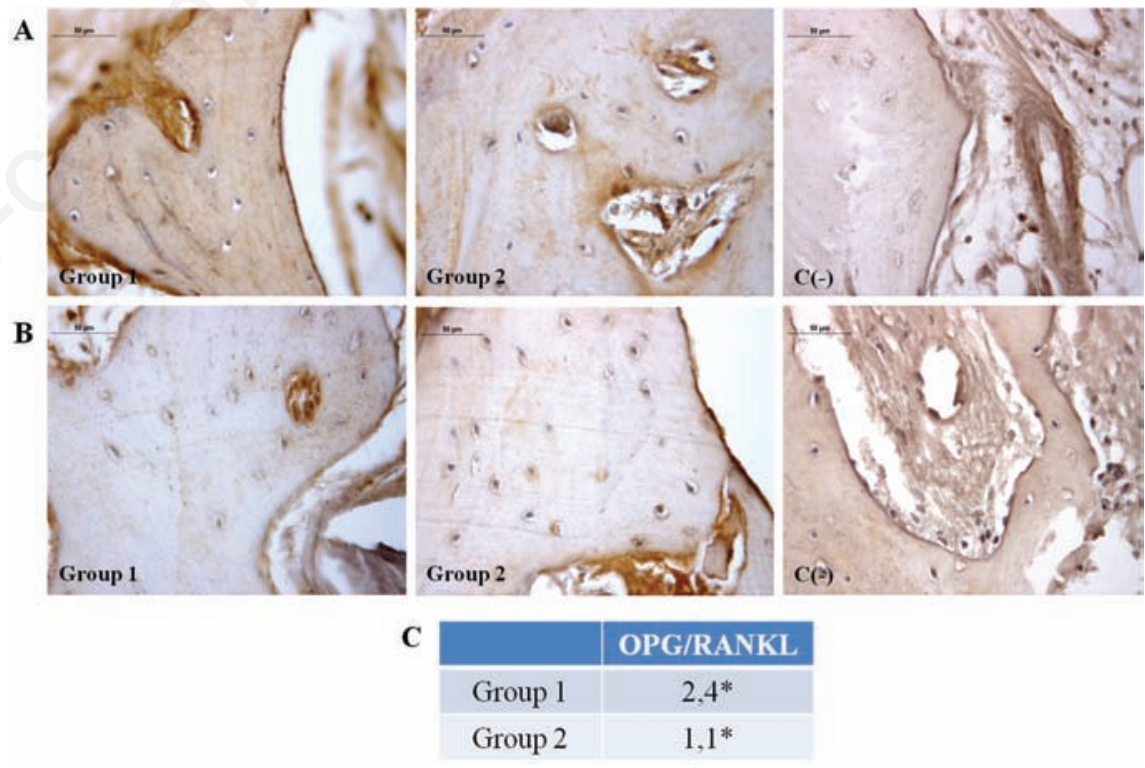

Figure 3. A) Immunohistochemical analysis of OPG expression in Group 1 and Group 2 specimens. Moderate OPG immunolabeling in Group 1 and weak OPG immunolabeling in Group 2 bone tissue; no OPG immunostaining is seen in negative control. B) Immunohistochemical analysis of RANKL expression, in Group 1 and Group 2 specimens, respectively. Magnification 40x. Group 1: bone tissue specimens obtained from equine-derived bone substitute grafted area; Group 2: bone tissue specimens obtained from calvaria bone grafted area; C(-), negative control. Weak RANKL immunolabeling in both Group 1 and Group 2 bone tissue; no RANKL immunostaining is seen in negative control. C) OPG/RANKL ratio values; ${ }^{*} \mathbf{P}<0.05$. 
the following grading: 0-0.2 (slight), 0.21-0.40 (fair), 0.41-0.60 (moderate), 0.61-0.80 (substantial), and 0.81-1.0 (almost perfect) ${ }^{20}$.

Negative control images were randomly chosen. The statistical significance of the results was evaluated by the Wilcoxon, Mann-Whitney Test, using R Software, ver. 2.12.1 for Mac and setting $\mathrm{P}=0.05$. After collecting results, the mean data were reported and showed in an histogram using Excel 2010 for Microsoft Windows.

\section{Results}

\section{Light microscopy analysis and immunohistochemistry}

Morphological analysis was performed at light microscope after hematoxylin-eosin staining (Figure 1). In Group 1 specimens the native bone tissue and connective tissue fibers could be identified. Moreover, the grafted biomaterial particles are easily distinguished (purple areas) due to their lack of tissue structure, as better shown by the inset. Group 2 sample shows large mineralized areas close to dense areas of connective tissue, within which newly formed bone is recognizable because of osteocyte lacunae lack and absence of lamellar organization (inset). Molecular modifications occurring after bone substitutes placement, concerning their ability to be integrated and to be clinically suitable, were then investigated by immunohistochemical analysis. TGF- $\beta 1$ expression, essential for osteoblastic differentiation and osteoid and extracellular proteins production, do not show any statistically significant difference between the two experimental groups (Figure 2); in fact, moderate TGF- $\beta 1$ immunolabeling is seen both in Group 1 and Group 2. Moreover, since TGF- $\beta 1$ stimulates OPG production, inhibiting the RANKL/RANK interaction, OPG and RANKL expressions were checked. Moderate OPG immunolabeling is found in Group 1 and weak OPG immunolabeling in Group 2, whereas weak RANKL immunolabeling is seen both in Group 1 and Group 2. The OPG/RANKL ratio is higher than 1 in both groups. However, Group 1 samples show a mean ratio of about four fold higher than that observed in Group 2 specimens ( 2.4 vs 1.1), due to the concomitant increase of OPG and a significant decrease of RANKL expression in samples from sites regenerated with the equinederived bone substitute (Figure 3 ). When the expression of VEGF, an angiogenic factor involved in early bone remodeling phases, was evaluated a very strong VEGF immunolabeling in Group 1 and strong VEGF immunolabeling in Group 2 is found (Figure 4) $(\mathrm{P}<0.05)$.

Finally, new bone formation and bone mineralizing processes were assessed taking into consideration BSP and SPARC expression levels, respectively. Weak BSP immunolabeling in
A
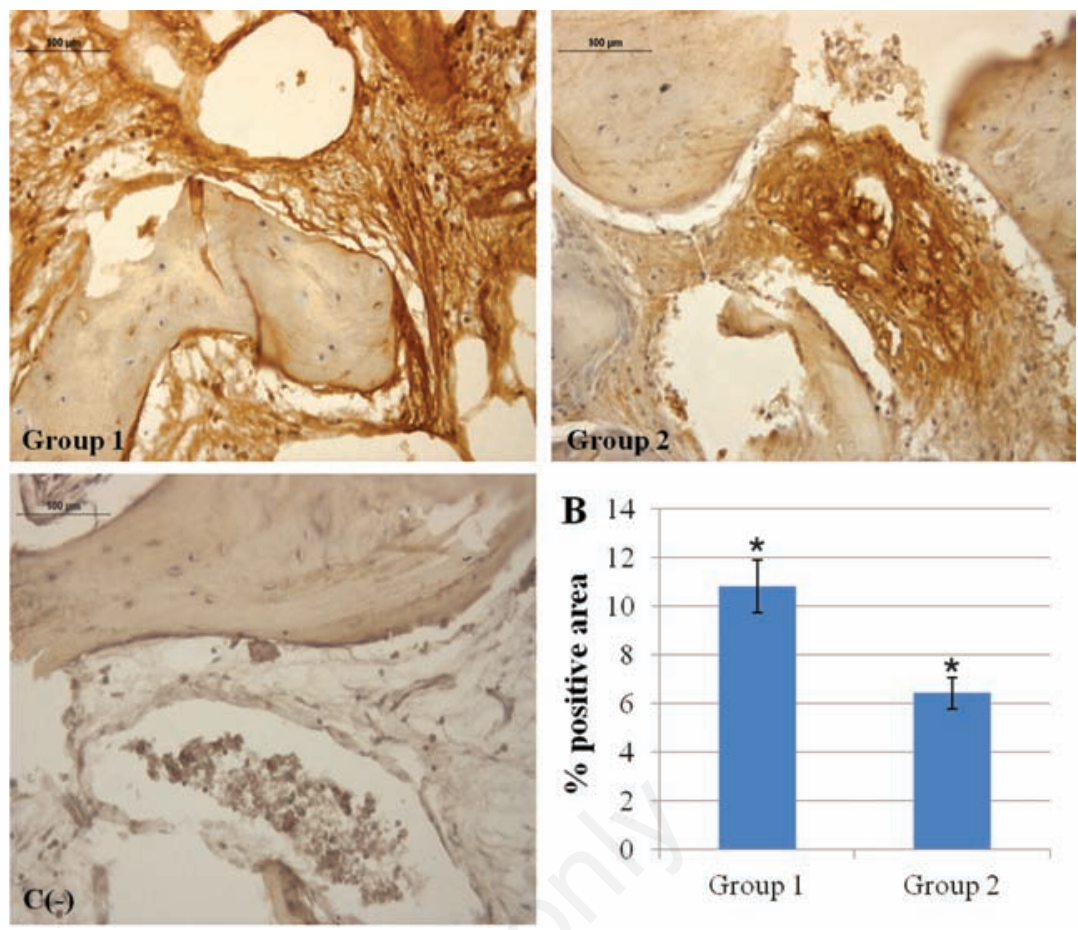

Figure 4. A) Immunohistochemical analysis of VEGF expression in Group 1 and Group 2 specimens. Magnification 20x. Group 1: bone tissue samples obtained from equinederived bone substitute grafted area; Group 2: bone tissue specimens obtained from calvaria bone grafted area; $C(-)$, negative control. Very strong VEGF immunolabeling in Group 1 and strong VEGF immunolabeling in Group 2 bone tissue; no VEGF immunostaining is seen in negative control. B) Graphic representation of densitometric analysis of VEGF positive area \pm SD determined by direct visual counting of ten fields (mean values) for each of five slides per specimens at $20 x$ magnification $\left({ }^{*} \mathrm{P}<0.05\right)$.

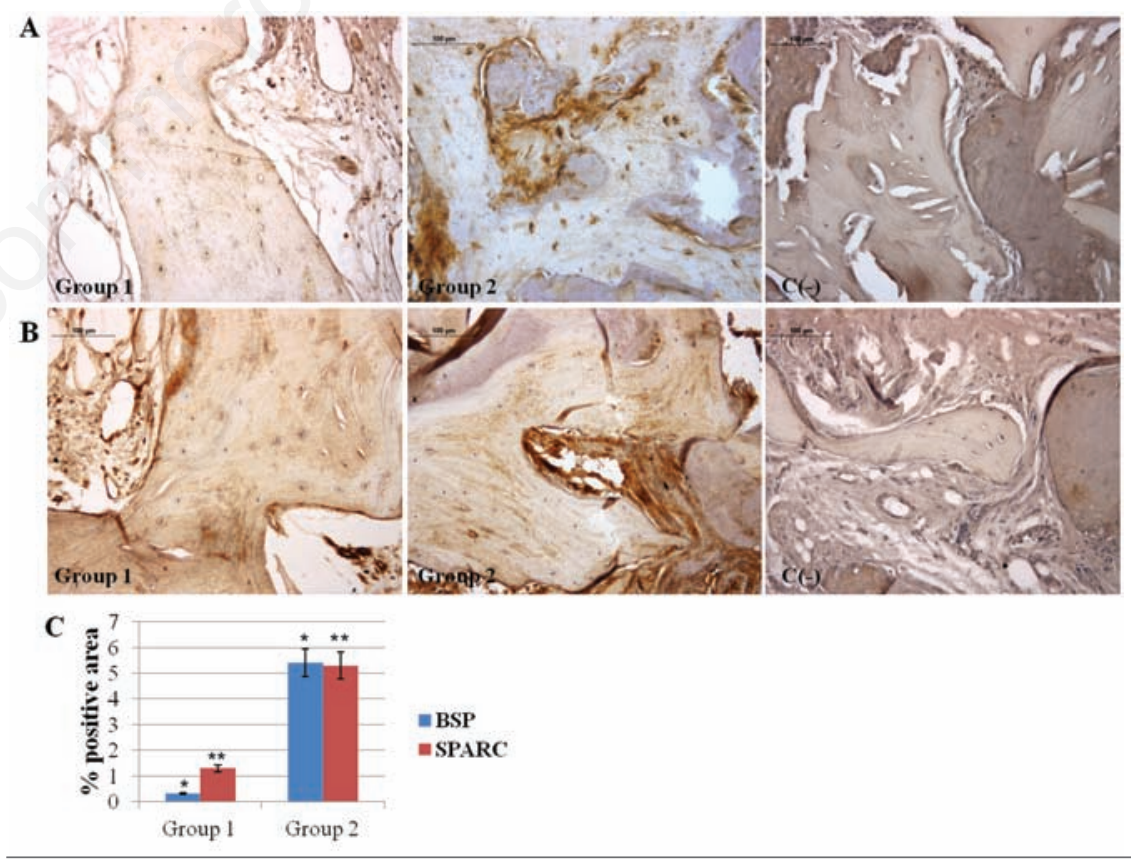

Figure 5. A) Immunohistochemical analysis of BSP expression in Group 1 and Group 2 specimens, respectively. Weak BSP immunolabeling in Group 1 and moderate BSP immunolabeling in Group 2 bone tissue; no BSP immunostaining is seen in negative control. B) Immunohistochemical analysis of SPARC expression, in Group 1 and Group 2 specimens, respectively. Magnification 20x. Group 1: bone tissue specimenss obtained from equinederived bone substitute grafted area; Group 2: bone tissue specimens obtained from calvaria bone grafted area; $\mathrm{C}(-)$, negative control. Weak SPARC immunolabeling in Group 1 and moderate SPARC immunolabeling in Group 2 bone tissue; no SPARC immunostaining is seen in negative control. C) Graphic representation of densitometric analysis of BSP and SPARC positive area \pm SD determined by direct visual counting of ten fields (mean values) for each of five slides per specimens at $20 \mathrm{x}$ magnification $\left({ }^{*} \mathrm{P}<0.001 ;{ }^{* *} \mathrm{P}<0.001\right)$. 
Group 1 and moderate BSP immunolabeling in Group 2 is seen, whereas weak SPARC immunolabeling in Group 1 and moderate SPARC immunolabeling in Group 2 is evidenced $(\mathrm{P}<0.001)$ (Figure 5). For all densitometric evaluations, interobserver agreement, measured using the Kappa coefficient, was 0.90 (almost perfect).

\section{Discussion}

Regenerative procedures employing autologous, homologous or heterologous bone grafts lead to bone defect repair through different biological mechanisms. The very good results obtained with the use of autologous bone grafts were widely demonstrated. ${ }^{21,22}$ Histological and clinical studies showed the possibility to have predictable results with the use of calvaria bone grafts, with no inflammatory phenomena and a minimum resorption rate during the healing period, along with a high rate of clinical success for the subsequent implant rehabilitation. ${ }^{23}$

Clinicians are constantly searching for a heterologous bone substitute that combines the osteo-regenerative features of autologous bone eliminating the limits imposed by the need for a second surgery. Most of the currently used heterologous grafts do not show ideal characteristics for bone regeneration such as osteogenic, osteoinductive and angiogenic potentials, biological safety, no size restrictions, long shelf life and reasonable cost. ${ }^{24}$

The present study aimed to evaluate, by morphological and immunohistochemical analyses, an equine bone substitute and an autologous bone graft, 6 months after the graft placement, in terms of ability of integration with the host tissue. The graft integration process is in part similar to bone fracture healing. ${ }^{8}$ Complete healing is obtained during the remodeling phase, when osteoblasts and osteoclasts cooperate to convert the callus of a fracture in a definitive functional bone structure. Bone resorption and formation events are tightly coordinated and balanced in healthy bone. ${ }^{25}$ For this reason, the first step of our study was to evaluate TGF $\beta 1$ expression, as its role in osteoblast/osteoclast coupling was demonstrated. ${ }^{10}$ Both Group 1 and Group 2 show a discrete TGF $\beta 1$ expression, suggesting that bone remodeling phenomena occurred in all the grafted areas. To better elucidate the host tissue response to the different bone substitutes, attention was focused on the OPG/RANKL ratio, often used as a bone resorption index. ${ }^{11}$ In fact, $\mathrm{OPG}$, by inhibiting the RANKL/RANK binding, protects osteoclastsmediated bone resorption acting on osteoclasts differentiation from precursors. ${ }^{8}$ Recent studies showed an increasing expression and activity of RANKL in bone pathologies charac- terized by bone resorption such as osteoporosis and osteoarthritis, and a compensatory increase of OPG in bone diseases, such as Paget's syndrome, in which an anomalous higher bone formation takes place. ${ }^{25}$

The balance between RANKL and OPG controls the osteoclast activity and undergoes an endocrine regulation. In fact, it is usually preferable to evaluate $\mathrm{OPG} / \mathrm{RANKL}$ ratio rather than OPG and RANKL absolute values. Thus an $0 \mathrm{PG} / \mathrm{RANKL}$ ratio $<1$ suggests a RANKL predominant activity, and, as a consequence, bone resorption events are predominant; on the other hand an 0PG/RANKL ratio >1 reveals OPG greater activity and predominant in new bone formation processes. ${ }^{26}$ The specimens of both groups show an OPG/RANKL ratio $>1$, suggesting that the bone protection process was predominant on RANKL/RANK cascade activation. However, the lower values of Group 2 specimen led us to hypothesize that the integration between host bone and autologous graft could be at a more advanced stage in respect to Group 1 specimens, since the occurrence of bone resorption and new bone apposition phenomena was nearer to balance in Group 2. This evidence is also confirmed by morphological analysis, that reveals a stronger integration between host tissue and bone graft in Group 2 specimens while, on the contrary, in Group 1 specimens some of the equine-derived biomaterial particles are still evident and few newly formed mineralized tissue areas can be observed.

In order to support these morphological aspects, angiogenesis, new bone formation and mineralization processes were evaluated, by investigating VEGF, BSP, and SPARC expression, respectively. Angiogenesis has a crucial role in the regulation of bone remodeling and repair. ${ }^{27}$. New blood vessel formation is essential to allow circulating osteoclast and osteoblast precursors to move towards the site of remodeling. ${ }^{28}$ Moreover, VEGF supports osteoblast growth in the initial phase of bone graft integration. ${ }^{13}$ In our study, VEGF expression is higher in Group 1 specimens indicating that intense neo-angiogenic phenomena were occurring in heterologous bone grafted sites, six months after the maxillary sinus augmentation procedure. This evidence could point to the fact that in autologous bone grafts this phase was already ended. In previous reports from our laboratory, in fact, extra-oral autologous bone grafts, showing an intense angiogenesis phenomena four months after grafting, with VEGF values significantly higher than in native bone tissue were found. ${ }^{29}$

Furthermore, the expression of molecules, such as BSP and SPARC, index of graft consolidation involved in new bone formation and mineralizing activity, indicate that sites treated with calvaria bone graft seemed to reach earlier a higher stage of mineralization compared to the equine bone grafted specimens.
Such results led us to conclude that host bone tissue, undergoing regenerative phenomena, positively reacted to the placement of both biomaterials. In particular, the equine-derived biomaterial shows good characteristics, in terms of both clinical and microscopic integration. However, at the same experimental time, sites treated with autologous bone clearly show a better organization, which could ensure a better primary stability to the implant and a higher predictability of the implant-prosthetic rehabilitation. In addition, in sites treated with autologous bone, a balance between matrix deposition and resorption is observed, suggesting that the regenerative process shows a higher rate of progression, than in sites treated with the equine bone graft in which this process seems to be slower.

\section{References}

1. Jensen OT, Shulman LB, Blocks MS, Iacono VJ. Report of the sinus consensus conference of 1996. Int J Oral Max Impl 1998;13:11-45.

2. Tetè S, Zara S, Vinci R, Zizzari V, Di Tore U, Mastrangelo F, et al. Vascular endothelial growth factor and e-nitric oxide synthase-mediated regenerative response occurring upon autologous and heterologous bone grafts. Int $\mathrm{J}$ Immunopathol Pharmacol 2009;22:1105-16.

3. Mangano C, Scarano A, Iezzi G, Orsini G, Perrotti V, Mangano F, et al. Maxillary sinus augmentation using an engineered porous hydroxyapatite : a clinical, histological, and transmission electron microscopy study in man. J Oral Implantol 2006;32: 122-31.

4. Hallman M, Lundgren S, Sennerby L. Histologic analysis of clinical biopsies taken 6 months and 3 years after maxillary sinus floor augmentation with $80 \%$ bovine hydroxyapatite and $20 \%$ autogenous bone mixed with fibrin glue. Clin Implant Dent R 2001;3:87-96.

5. Mordenfeld A, Hallman M, Johansson CB, Albrektsson T. Histological and histomorphometrical analyses of biopsies harvested 11 years after maxillary sinus floor augmentation with deproteinized bovine and autogenous bone. Clin Oral Implan Res 2010;21:961-70.

6. Di Stefano DA, Artese L, Iezzi G, Piattelli A, Pagnutti S, Piccirilli M, et al. Alveolar ridge regeneration with equine spongy bone: a clinical, histological, and immunohistochemical case series. Clin Implant Dent $\mathbf{R}$ 2009;11:90-100.

7. Perrotti V, Nicholls BM, Piattelli A. Human osteoclast formation and activity on an equine spongy bone substitute. Clin Oral Implan Res 2009;20:17-23. 
8. Dimitriou R, Jone, E, McGonagle D, Giannoudis PV. Bone regeneration: current concepts and future directions. BMC Med 2011;9:66.

9. Lieberman JR, Daluiski A, Einhorn TA. The role of growth factors in the repair of bone. Biology and clinical applications. J Bone Joint Surg Am 2002; 84: 1032-1044.

10. Quinn JMW, Itoh K, Udagawa N, Hausler $\mathrm{K}$, Yasuda H, Shima N, et al. Transforming growth factor $\beta$ affects osteoclast differentiation via direct and indirect actions. $\mathrm{J}$ Bone Min Res 2001;16:1787-94.

11. Trouvin AP, Goëb V. Receptor activator of nuclear factor-кB ligand and osteoprotegerin: maintaining the balance to prevent bone loss. Clin Interv Aging 2010;5:345-54

12. Khosla S. Minireview: the OPG/RANKL/ RANK system. Endocrinology 2001;142: 5050-55.

13. Gerber HP, Vu TH, Ryan AM, Kowalski J, Werb Z, Ferrara N. VEGF couples hypertrophic cartilage remodeling, ossification and angiogenesis during endochondral bone formation. Nat Med 1999;5:623-28.

14. Raynal C, Delmas PD, Chenu C. Bone sialoprotein stimulates in vitro bone resorption. Endocrinology 1996;137:23472354.

15. Malaval L, Wade-Guéye NM, Boudiffa M, Fei J, Zirngibl R, Chen F, et al. Bone sialoprotein plays a functional role in bone formation and osteoclastogenesis. J Exp Med 2008;205:1145-53.

16. Boudiffa M, Wade-Gueye NM, Guignandon A, Vanden-Bossche A, Sabido 0, Aubin JE, et al. Bone sialoprotein deficiency impairs osteoclastogenesis and mineral resorption in vitro. J Bone Min Res 2010;25:2669-79.

17. Alford AI, Hankenson KD. Matricellular proteins: Extracellular modulators of bone development, remodeling, and regeneration. Bone 2006;38:749-57.

18. Misch CE, Chiapasco M, Jensen OT. Indications for and Classification of Sinus Bone Grafts. In: Jensen OT, ed. The Sinus Bone Graft. Quintessence Pubblishing Co, 2006.

19. Musumeci G, Loreto C, Carnazza ML, Coppolino F, Cardile V, Leonardi R. Lubricin is expressed in chondrocytes derived from osteoarthritic cartilage encapsulated in poly (ethylene glycol) diacrylate scaffold. Eur J Histochem. 2011; 55:e31.

20. Musumeci G, Carnazza ML, Loreto C, Leonardi R, Loreto C. $\beta$-defensin-4 (HBD4) is expressed in chondrocytes derived from normal and osteoarthritic cartilage encapsulated in PEGDA scaffold. Acta Histochem. 2012;114:805-12.

21. Crespi R, Vinci R, Capparè $P$, Gherlone $E$, Romanos GE. Calvarial versus iliac crest for autologous bone graft material for a sinus lift procedure: a histomorphometric study. Int J Oral Max Impl 2007;22:527-32.

22. Gutta R, Waite PD. Outcomes of calvarial bone grafting for alveolar ridge reconstruction. Int J Oral Max Impl 2009;24:131 36 .

23. Tetè S, Vinci R, Zara S, Zizzari V, Cataldi A, Mastrangelo F, et al. Atrophic jaw reconstruction by means of calvarial bone graft: long-term results. J Craniofac Surg 2010;
21:1147-52.

24. Fröhlich M, Grayson WL, Wan LQ, Marolt D, Drobnic M, Vunjak-Novakovic G. Tissue engineered bone grafts: biological requirements, tissue culture and clinical relevance. Curr Stem Cell Res Ther 2008;3: 254-64.

25. Alvarez L, Peris P, Guanabens N, Vidal S, Ros I, Pons F, et al. Serum osteoprotegerin and its ligand in Paget's disease of bone: relationship to disease activity and effect of treatment with bisphosphonates. Arthritis Rheum-US 2003;48:824-28.

26. Fonseca JE, Cortez-Dias N, Francisco A, Sobral M, Canhão H, Resende C, et al. Inflammatory cell infiltrate and RANKL/ OPG expression in rheumatoid synovium: comparison with other inflammatory arthropathies and correlation with outcome. Clin Exp Rheumatol 2005;23: 18592.

27. Gerstenfeld LC, Cullinane DM, Barnes GL, Graves DT, Einhorn TA. Fracture healing as a post-natal developmental process: molecular, spatial, and temporal aspects of its regulation. J Cell Biochem 2003;88:87384.

28. van Oers RF, Ruimerman R, Tanck E, Hilbers PA, Huiskes R. A unified theory for osteonal and hemi-osteonal remodeling. Bone 2008;42:250-59.

29. Tetè $S$, Vinci R, Zara S, Zizzari V, De Carlo A, Falco G, et al. Long-term evaluation of maxillary reconstruction by iliac bone graft. J Craniofac Surg 2011;22:1702-7. 